\title{
Ethnobotanical Survey of Plants Commonly used for Diabetes in Tarlac of Central Luzon Philippines
}

\author{
Erwin C. Mina, ${ }^{a, b}$ Josefina F. Minac \\ ${ }^{a}$ Faculty of Natural Sciences, College of Science, Tarlac State University, Tarlac City, Philippines \\ ${ }^{b}$ Natural Products Research Center, Tarlac State University, Tarlac City, Philippines \\ 'San Nicolas Elementary School, Tarlac City School Division, Tarlac City, Philippines
}

\begin{abstract}
Introduction: Plants have been used for the treatment of diabetes in Philippine system of medicine and in other countries. They provide clues for the development of new and better oral drugs for diabetes due to the fact that prevalence of diabetes is on a steady upsurge worldwide and identified as one of the leading causes of mortality in Philippines. Methods: An ethnobotanical survey was carried out in Tarlac of Central Luzon Philippines to evaluate the relative efficacy of the plants used to treat diabetes. A total number of 450 respondents medically diagnosed of having diabetes were interviewed using a previously prepared questionnaire. Fidelity levels (FLs) and use values (UVs) were calculated to identify and verify most preferred plant species used in study areas. Results: A total of 25 plant species are utilized by the respondents to treat diabetes. Medicinal plants commonly utilized are Momordica charantia Linn., Moringa oleifera Lam, Annona muricata Linn., Psidium guajava Linn., Lagerstroemia speciosa (L.) Pers., Hibiscus esculentis Linn., Amomum zingiber Linn., Myrtus cumini Linn., Garcinia mangostana Linn., and Aloe vera Linn. Decoction process is used to prepare the leaves of the medicinal plants for medical consumption in the form of tea. Conclusion: This study confirms the use of different plant species to treat diabetes. It further affirms that most people with diabetes in the study areas rely on traditional medicine for their primary health care needs.
\end{abstract}

KEYWORDS: diabetes, survey, ethnobotanical, ethnomedicine, Philippines

\section{INTRODUCTION}

Plant with medicinal values is regarded as the oldest form of healthcare known to mankind. Plants such as herbs, spices, shrubs, grasses, trees had been used by all cultures in every walks of life throughout history. ${ }^{1-2}$ Herbal medicine was an integral part of the development of modern civilization. Indeed, by nature, man observed and appreciated the great diversity of plants available that surround him. Plants provided food, clothing, shelter, fuel and medicine. Much of the medicinal use of plants seems to have been developed through observations of wild animals, and by trial and error. Men of every tribe added the medicinal power of herbs in their area to its knowledge base. ${ }^{3}$ They meticulously gathered information on plants and developed well-defined herbal pharmacopoeias. Apparently, well into the

Corresponding author:

Erwin C. Mina

Faculty of Natural Sciences,

College of Science,

Tarlac State University,

Tarlac City, Philippines

Email address: mina_erwin41@yahoo.com /

erwin.mina@tsu.edu.ph $20^{\text {th }}$ century much of the pharmacopoeia was derived from the herbal lore of native peoples. Many drugs commonly used today are of herbal origin. $^{4}$

Diabetes is a disorder of carbohydrate, fat and protein metabolism attributed to diminished production of insulin or mounting resistance to its action. The prevalence of diabetes is on a steady increase worldwide and it is now identified as one of the major threats to human health in the $21^{\text {st }}$ century. According to the Department of Health, diabetes mellitus is one of the major causes of death in Region III. $^{5}$ Early Philippine material medica started to appear only during the Spanish period. A notable development of the Galleon Trade was the cultural interchange between the two colonies not only in terms of words but even in trade and commerce; plants also were being part of such exchange. ${ }^{6}$

Plants have been used in treatment of diabetes all over the world for centuries. Wide variety of plant derived active principles representing numerous classes of chemical compounds have shown potential for the use in treatment of diabetes. Among the classes of chemical compounds isolated from plants with documented biological activity are 
alkaloids, glycosides, galactomannan, gum, peptidoglycan, glycopeptide, amino acids and inorganic ions. ${ }^{7-8}$

In view of an increasing interest to fight diabetes, this study was undertaken to identify the medicinal plants commonly utilized by the people with diabetes for treating the ailment in the province of Tarlac. It examined the frequency of medications used by the people in treating diabetes. Likely, it determined the manner of how medicinal plants are prepared for consumption.

\section{METHODOLOGY}

The study utilized a descriptive method of research. It identified and determined the most common and popularly used medicinal plant species utilized by the people with diabetes in the province of Tarlac. Tarlac is a landlocked province of the Philippines with a land area of 2,736.6 square kilometers with a population of $1,273,240$ as of $2010^{9}$ located in the Luzon Island. Its capital is Tarlac City.

The province is situated at the center of the central plains of Luzon consisting of 17 municipalities, landlocked by four provinces: Pampanga on the south, Nueva Ecija on the east, Pangasinan on the north, and Zambales on the west. It lies $15.4667^{\circ} \mathrm{N}$ and $120.5833^{\circ} \mathrm{E}$. Approximately $75 \%$ of the province is plain while the rest is hilly to mountainous. Like the rest of Central Luzon, the province has two distinct seasons: dry from November to April and wet for the rest of the year. It is a part of Central Luzon, which is composed of Aurora, Bataan, Bulacan, Nueva Ecija, Pampanga, Tarlac, and Zambales provinces respectively.

Four hundred fifty (450) respondents with diabetes (regardless of diabetes classification) were interviewed from different municipalities of the province. A written consent was given to them before the activity. Respondents were asked or wrote the plants cronologically with medicinal values for the treatment of diabetes in a tabular form as can be seen in Table III. People with diabetes and with age ranges from 40 - 90 years old was included in the study. A voucher specimen of the identified medicinal plants used in the treatment of diabetes consisting of leaves, stem, flowers and fruits was brought to UST Herbarium, University of Santo Tomas for taxonomic verification.

Fidelity level $(\mathrm{FL})^{10}$ is calculated to determine the most frequently used medicinal plant species for treating diabetes while use value (UV) ${ }^{11}$ for species is calculated to find out the relative importance of a plant species used as medicine in the study areas. Towards this end, the fidelity level of each plant was determined as follows: $\mathrm{FL}=(/ \mathrm{p} / / \mathrm{u}) \times 100$. Ip is the number of respondents who independently mentioned the use of a plant species for the treatment of diabetes; and $/ u$ is the total number of respondents who mentioned the plant species. The relative importance of each plant species known locally to be used as herbal remedy is reported as use value (UV) and it was calculated using the following formula: $U V=\Sigma U / n$. $U$ is the number of use reports cited by each respondent for a given plant species and $n$ is the total number of respondents interviewed for a given plant. The UV is helpful in determining the plants with the highest use (most frequently indicated) in the treatment of an ailment.

\section{RESULTS AND DISCUSSION}

In the Philippines the history of botany started from two periods namely, Pre-Linnaean, previous to the publication of the Species Plantarum by Linnaeus in 1753, and Post-Linnaean. Plant myths and legends abound in Philippine Folklore. ${ }^{12}$ Although no written herbal lore has been handed down from ancestors, but the practice of using herbs coupled with rituals is well known thru oral records being told from generations to generations where herbolarios are the instrument of communication.

Four hundred fifty respondents (215 male and 235 female individuals, aged 40 to 90 years) medically diagnosed of having diabetes participated in the study. They were selected through purposive method from 17 municipalities (Anao, Bamban, Camiling, Capas, Concepcion, Gerona, Lapaz, Mayantoc, Moncada, Paniqui, Pura, Ramos, San Clemente, Sta. Ignacia, San Manuel, and Tarlac City, Victoria) of Tarlac province. Respondents are mostly coming from selected barangays of every municipality.

Twenty five plant species of twenty one families were mentioned by 450 respondents to treat diabetes. Table I and II confirm the findings of the World Health Organization (WHO) which estimates that eighty percent $(80 \%)$ of the people living in developing countries of the world rely on traditional or folkloric medicine for their primary health care needs. ${ }^{13-16}$

About eighty five percent (85\%) of traditional or folkloric medicine involves the use of plant extracts to cure identified ailments. ${ }^{17-23}$ In line with the upsurge significance of traditional medicine in various healthcare systems around the world, the WHO Traditional Medicine Strategy was developed and launched in response to the World Health Assembly resolution on traditional medicine. The strategy aims to support Member States in developing proactive policies and implementing action plans that will strengthen the role traditional medicine plays in keeping populations healthy. ${ }^{24}$ 
Table I: Top ten plants used for diabetes in the province of Tarlac

\begin{tabular}{|c|c|c|c|c|c|}
\hline Rank & $\begin{array}{l}\text { Common Name } \\
\text { (Family) }\end{array}$ & Scientific Name & English Name & Other Names & $\mathrm{FL}^{\mathrm{a}}$ \\
\hline 1 & $\begin{array}{l}\text { Ampalaya } \\
\text { (Cucrbitaceae) }\end{array}$ & $\begin{array}{l}\text { Momordica charantia Linn. } \\
\text { Mormodica cylindrica Blanco } \\
\text { Mormodica chinensis Spreng } \\
\text { Mormodica balsamina Blanco } \\
\text { Mormodica muricata Willd. }\end{array}$ & $\begin{array}{l}\text { Balsam apple } \\
\text { Bitter gourd } \\
\text { Bitter melon } \\
\text { Balsam pear }\end{array}$ & $\begin{array}{l}\text { Apalia } \\
\text { Apapet } \\
\text { Margoso } \\
\text { Paria }\end{array}$ & 100.0 \\
\hline 2 & $\begin{array}{l}\text { Malunggay } \\
\text { (Moringaceae) }\end{array}$ & $\begin{array}{l}\text { Moringa oleifera Lam. } \\
\text { Moringa nux-ben Perr. } \\
\text { Moringa pterygosperma Gaertn. } \\
\text { Guilandina moringa Linn. }\end{array}$ & $\begin{array}{l}\text { Drumstick tree } \\
\text { Horse-radish tree } \\
\text { Miracle tree }\end{array}$ & $\begin{array}{l}\text { Arunggai } \\
\text { Kalamungai } \\
\text { Maruñgaai }\end{array}$ & 90.67 \\
\hline 3 & $\begin{array}{l}\text { Guyabano } \\
\text { (Anonaceae }\end{array}$ & $\begin{array}{l}\text { Annona muricata Linn. } \\
\text { Annona macrocarpa Werkle } \\
\text { Annona bonplandiana Kunth } \\
\text { Annona cearensis Barb. Rodr. } \\
\text { Guanabanus muricatus M. Gomez }\end{array}$ & $\begin{array}{l}\text { Graviola } \\
\text { Prickly custard apple } \\
\text { Soursop }\end{array}$ & $\begin{array}{l}\text { Bayubana } \\
\text { Guayabano }\end{array}$ & 88.89 \\
\hline 4 & $\begin{array}{l}\text { Bayabas } \\
\text { (Myrtaceae) }\end{array}$ & $\begin{array}{l}\text { Psidium guajava Linn } \\
\text { Psidium cujavus Linn. } \\
\text { Psidium aromaticum Blanco } \\
\text { Guajava pyrifera (l.) Kuntz. }\end{array}$ & Guava & $\begin{array}{l}\text { Bagabas } \\
\text { Bayauas } \\
\text { Guyabas } \\
\text { Tayabas }\end{array}$ & 83.33 \\
\hline 5 & $\begin{array}{l}\text { Banaba } \\
\text { (Lythraceae) }\end{array}$ & $\begin{array}{l}\text { Lagerstroemia speciosa (L.) Pers. } \\
\text { Lagerstroemia reginae Roxb. } \\
\text { Lagerstroemia flos-reginae Retz. } \\
\text { Munchausia speciosa (L.) }\end{array}$ & $\begin{array}{l}\text { Pride of India } \\
\text { Queen's flower }\end{array}$ & $\begin{array}{l}\text { Makablos } \\
\text { Mitla } \\
\text { Nabulong } \\
\text { Tabangau }\end{array}$ & 66.67 \\
\hline 6 & $\begin{array}{l}\text { Okra } \\
\text { (Malvaceae) }\end{array}$ & $\begin{array}{l}\text { Abelmoschus esculentus (Linn.) } \\
\text { Moench. } \\
\text { Hibiscus esculentis Linn. }\end{array}$ & Lady fingers & Gumbo & 66.62 \\
\hline 7 & $\begin{array}{l}\text { Luya } \\
\text { (Zingirberaceae) }\end{array}$ & $\begin{array}{l}\text { Amomum zingiber Linn. } \\
\text { Zingiber blancoi Hassk. } \\
\text { Zingiber officinale Roscoe }\end{array}$ & Ginger & $\begin{array}{l}\text { Agat } \\
\text { Baseng } \\
\text { Laya }\end{array}$ & 65.53 \\
\hline 8 & $\begin{array}{l}\text { Duhat } \\
\text { (Myrtaceaea) }\end{array}$ & $\begin{array}{l}\text { Calyptranthes jambolana Willd. } \\
\text { Eugenia cumini (Linn.) Druce } \\
\text { Myrtus cumini Linn. } \\
\text { Syzygium cumini Skeels }\end{array}$ & $\begin{array}{l}\text { Black plum } \\
\text { Indian blackberry } \\
\text { Java plum }\end{array}$ & $\begin{array}{l}\text { Duat } \\
\text { Lomboi } \\
\text { Longboi }\end{array}$ & 64.44 \\
\hline 9 & $\begin{array}{l}\text { Mangosteen } \\
\text { (Guttiferae) }\end{array}$ & $\begin{array}{l}\text { Garcinia mangostana Linn. } \\
\text { Mangostana garcinia Gaertn. }\end{array}$ & $\begin{array}{l}\text { King's fruit } \\
\text { Queen of Fruits }\end{array}$ & $\begin{array}{l}\text { Kanabla } \\
\text { Mangostan }\end{array}$ & 64.42 \\
\hline 10 & $\begin{array}{l}\text { Sabila } \\
\text { (Liliaceae) }\end{array}$ & $\begin{array}{l}\text { Aloe barbadensis Mill. } \\
\text { Aloe humilis Blanco } \\
\text { Aloe vera Linn. } \\
\text { Aloe perfoliata Linn. }\end{array}$ & $\begin{array}{l}\text { Aloe vera } \\
\text { Burn plant }\end{array}$ & $\begin{array}{l}\text { Acibar } \\
\text { Dilang buaya } \\
\text { Sabila pinya }\end{array}$ & 62.44 \\
\hline
\end{tabular}

${ }^{a}$ Fidelity level=[number of informants who independently utilized the use of a species for treating $\mathrm{db}(I \mathrm{l}) /$ total number of informants $(/ \mathrm{u})] \times 100$.

$\mathrm{FL}=(/ \mathrm{p} / / \mathrm{u}) \times 100$.

Figure 1 shows that the highest percentage distribution of respondents using plants as alternative health care for diabetes is 61-63 years old with $15.37 \%$ followed by $52-54$ years old with $12.96 \%$ respectively.

The most commonly utilized plant (Table I and Table III) was Momordica charantia Linn. with $100.0 \%$ fidelity level and 25 use reports by 28 respondents giving the highest use value of 0.89 . The plant is well appraised by all respondents as an antidiabetic plant. M.charantia Linn. is a yearround vegetable, extensively cultivated in the Philippines for its bitter edible fruit which is usually found in an open fields, thickets, and waste placebs at low and medium altitudes. ${ }^{1}$
Other important plants with high fidelity level and use value were Moringa oleifera Lam (FL of $90.67 \%$ and 20 use reports by 26 respondents with a UV of 0.77), Annona muricata Linn. (FL of $88.89 \%$ and 18 use reports by 24 respondents with a UV of 0.75 ), Psidium guajava Linn. (FL of $83.33 \%$ and 17 use reports by 23 respondents with a UV of 0.74 ), Lagerstroemia speciosa (L.) Pers. (FL of $66.67 \%$ and 16 use reports by 22 respondents with a UV of 0.73 ), Hibiscus esculentis Linn. (FL of $66.62 \%$ and 14 use reports by 21 respondents with a UV of 0.67 ), Amomum zingiber Linn. (FL of $65.53 \%$ and 13 use reports by 20 respondents with a UV of 0.65 ), Myrtus cumini Linn. and Garcinia mangostana Linn.

FL of $64.44 \%$ and 12 use reports by 19 respondents with a UV of 0.63 ), and Aloe vera Linn. (FL of $62.44 \%$ and 10 use reports by 15 respondents with a UV of 0.62). 
Table II: Occasionally used plants for diabetes

\begin{tabular}{|c|c|c|c|c|c|}
\hline Rank & $\begin{array}{l}\text { Common } \\
\text { Name }\end{array}$ & Scientific Name & English Name & Other Name & $\mathrm{FL}^{\mathrm{a}}$ \\
\hline 11 & $\begin{array}{l}\text { Abukado } \\
\text { (Lauraceae) }\end{array}$ & $\begin{array}{l}\text { Persea gratissima Gaertn. } \\
\text { Persea americana Mill. }\end{array}$ & $\begin{array}{l}\text { Avocado } \\
\text { Alligator pear }\end{array}$ & Abokado & 61.11 \\
\hline 12 & $\begin{array}{l}\text { Akapulko } \\
\text { (Fabaceae) }\end{array}$ & $\begin{array}{l}\text { Cassia alata Linn. } \\
\text { Herpetic alata Raf. }\end{array}$ & $\begin{array}{l}\text { Candle bush } \\
\text { Ringworm bush }\end{array}$ & $\begin{array}{l}\text { Andadasi-a-dadakdel } \\
\text { Bayabasin }\end{array}$ & 60.00 \\
\hline 13 & $\begin{array}{l}\text { Balbas pusa } \\
\text { (Lamiaceae) }\end{array}$ & $\begin{array}{l}\text { Orthosiphon aristatus (Blume) Miq. } \\
\text { Ocimum aristatum Blume }\end{array}$ & $\begin{array}{l}\text { Kidney tea } \\
\text { Cat's whisker }\end{array}$ & $\begin{array}{l}\text { Kabling-gubat } \\
\text { Kabling-parang }\end{array}$ & 59.56 \\
\hline 14 & $\begin{array}{l}\text { Balanaoi } \\
\text { (Labiatae) }\end{array}$ & $\begin{array}{l}\text { Ocimum album Blanco } \\
\text { Ocimum sanctum Linn. } \\
\text { Ocimum tenuiflorum L. }\end{array}$ & $\begin{array}{l}\text { Holy basil } \\
\text { Sacred basil }\end{array}$ & $\begin{array}{l}\text { Bidai } \\
\text { Sulasi } \\
\text { Kamangi }\end{array}$ & 58.89 \\
\hline 15 & $\begin{array}{l}\text { Bawang } \\
\text { (Liliaceae) }\end{array}$ & $\begin{array}{l}\text { Allium sativum Linn. } \\
\text { Allium pekinense Prokhanov }\end{array}$ & $\begin{array}{l}\text { Garlic } \\
\text { Nectar of the } \\
\text { Gods }\end{array}$ & $\begin{array}{l}\text { Bauang } \\
\text { Ahos }\end{array}$ & 58.44 \\
\hline 16 & $\begin{array}{l}\text { Caimito } \\
\text { (Sapotaceae) }\end{array}$ & $\begin{array}{l}\text { Chrysophyllum cainito Linn. } \\
\text { Calophyllum inophyllum Linn. }\end{array}$ & $\begin{array}{l}\text { Star apple } \\
\text { Star plum }\end{array}$ & Caymito & 57.78 \\
\hline 17 & $\begin{array}{l}\text { Dalanghita } \\
\text { (Rutaceae) }\end{array}$ & $\begin{array}{l}\text { Citrus aurantium L. var. mandarinum } \\
\text { Citrus madurrensis } \\
\text { Citrus reticulata Blanco } \\
\text { Citrus webberi Wester }\end{array}$ & $\begin{array}{l}\text { Mandarin orange } \\
\text { King orange }\end{array}$ & $\begin{array}{l}\text { Alsem } \\
\text { Ransas } \\
\text { Darañgita }\end{array}$ & 55.56 \\
\hline 18 & $\begin{array}{l}\text { Kalingag } \\
\text { (Lauraceae) }\end{array}$ & $\begin{array}{l}\text { Cinamomum mercadoi Vidal } \\
\text { Cinamomum zeylanicum F.-Vill. }\end{array}$ & Kalingag tree & $\begin{array}{l}\text { Kandoroma } \\
\text { Samiling }\end{array}$ & 54.44 \\
\hline 19 & $\begin{array}{l}\text { Kamatsile } \\
\text { (Leguminosae) }\end{array}$ & $\begin{array}{l}\text { Pithecellobium dulce (Roxb.) Benth. } \\
\text { Feuilleea dulcis Roxb. } \\
\text { Inga camatchili Perr. } \\
\text { Mimosa dulcis Roxb. }\end{array}$ & $\begin{array}{l}\text { Black bead } \\
\text { Monkeypod } \\
\text { Madras thorn }\end{array}$ & $\begin{array}{l}\text { Camachile } \\
\text { Damortis } \\
\text { Kamachili } \\
\text { Kamunsil }\end{array}$ & 52.22 \\
\hline 20 & $\begin{array}{l}\text { Kasuy } \\
\text { (Anacardiaceae } \\
\text { ) }\end{array}$ & $\begin{array}{l}\text { Anacardium occidentale Linn. } \\
\text { Acajuba occidentalis (L.) Gaertn. } \\
\text { Cassuvium pomiferum Lam. }\end{array}$ & Cashew & $\begin{array}{l}\text { Balubad } \\
\text { Sambalduke } \\
\text { Kasul }\end{array}$ & 51.33 \\
\hline 21 & $\begin{array}{l}\text { Lagundi } \\
\text { (Verbanaceae) }\end{array}$ & $\begin{array}{l}\text { Vitex negundo Linn } \\
\text { Vitex leucoxylon Blanco } \\
\text { Vitex paniculata Lam. }\end{array}$ & $\begin{array}{l}\text { Chinese chaste- } \\
\text { tree } \\
\text { Chinese chaste- }\end{array}$ & $\begin{array}{l}\text { Dangla } \\
\text { Dabtan } \\
\text { Sagarai }\end{array}$ & 46.22 \\
\hline 22 & $\begin{array}{l}\text { Mankit } \\
\text { (Rubiaceae) }\end{array}$ & $\begin{array}{l}\text { Rubia cordifolia Linn. } \\
\text { Rubia tinctorum L. var. cordifolia L. }\end{array}$ & $\begin{array}{l}\text { Heart-leaved } \\
\text { madder } \\
\text { Indian madder }\end{array}$ & $\begin{array}{l}\text { Kamagut } \\
\text { Patudtud }\end{array}$ & 42.89 \\
\hline 23 & Screw-pine & Pandanus odoratissimus Linn. f. & Screw-pine & Pandan lalaki & 42.22 \\
\hline 24 & Saging & Musa paradisiaca Linn. & Banana & Kela & 41.11 \\
\hline 25 & $\begin{array}{l}\text { Sinta } \\
\text { (Aranthaceae }\end{array}$ & $\begin{array}{l}\text { Andrographis paniculata (Burm. f.) } \\
\text { Wall. ex Nees } \\
\text { Justicia paniculata }\end{array}$ & $\begin{array}{l}\text { King of bitters } \\
\text { Chiretta }\end{array}$ & $\begin{array}{l}\text { Likha } \\
\text { Serpentina }\end{array}$ & 40.67 \\
\hline
\end{tabular}

${ }^{a}$ Fidelity leve $=[$ number of informants who independently utilized the use of a species for treating $\mathrm{db}(I \mathrm{p}) /$ total number of informants $(/ \mathrm{u})] \times 100$. $\mathrm{FL}=(/ \mathrm{p} / / \mathrm{u}) \times 100$.

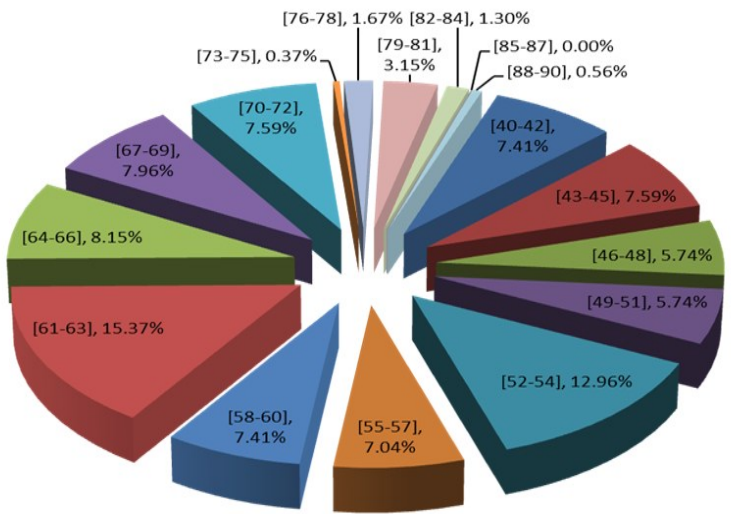

Figure 1. Percentage distribution of respondents at different age brackets 
Most of these plants are abundant in the study areas. ${ }^{25}$ The plant with the lowest fidelity level (40.67\%) and use value (0.06) is Andrographis paniculata (Burm. f.) Wall. ex Nees by 18 respondents but only one use report. The respondents are using the plant since they are diagnosed with diabetes. Nevertheless, the following plants such Persea gratissima Gaertn., Cassia alata Linn., Orthosiphon aristatus (Blume) Miq., Ocimum album Blanco, Allium sativum Linn., Chrysophyllum cainito Linn., Citrus aurantium L. var. mandarinum, Cinamomum mercadoi Vidal, Pithecellobium dulce (Roxb.) Benth., Anacardium occidentale Linn., Vitex negundo Linn, Rubia cordifolia Linn., Pandanus odoratissimus Linn. f.,
Musa paradisiaca Linn. and Andrographis paniculata (Burm. f.) Wall. ex Nees were great of help in the treatment of diabetes as per testified by the respondents in the study areas. UVs are high when there are many use-reports for a plant and low when there are few reports related to its use.

Finally, as can be seen from Table III, the leaf is considered most as the part of the plant being utilized in the treatment of diabetes. The leaves are prepared either by decoction or extraction and mostly are taken as a form of tea, once a day (1D), twice a day (2D) or thrice a day (3D). Some of these pants are also consumed as vegetables and eaten fresh by the respondents.

Table III: Preparation of the plants for consumption

\begin{tabular}{|c|c|c|c|c|c|c|c|c|}
\hline Rank & $\begin{array}{c}\text { Name of } \\
\text { plant }\end{array}$ & $\begin{array}{c}\text { Part } / \mathbf{s} \\
\text { used }\end{array}$ & Preparation & Administration & Dosage $^{a}$ & $U$ & $n$ & $U^{b}$ \\
\hline \multirow{4}{*}{1} & \multirow{4}{*}{ Ampalaya } & Leaves & Decoction & Tea & 1D & \multirow{4}{*}{25} & \multirow{4}{*}{28} & \multirow{4}{*}{0.89} \\
\hline & & Fruits & Decoction & Viand & $1 \mathrm{D}$ & & & \\
\hline & & Flowers & Decoction & Tea & $1 \mathrm{D}$ & & & \\
\hline & & Seeds & Extraction & Tea & 1D & & & \\
\hline 2 & Malunggay & Leaves & Decoction & Tea / Viand & $2 \mathrm{D}$ & 20 & 26 & 0.77 \\
\hline \multirow{2}{*}{3} & \multirow{2}{*}{ Guyabano } & Leaves & Decoction & Tea & 1D & \multirow{2}{*}{18} & \multirow{2}{*}{24} & \multirow{2}{*}{0.75} \\
\hline & & Fruit & Fresh & Eaten as raw & 1D & & & \\
\hline \multirow{2}{*}{4} & \multirow{2}{*}{ Bayabas } & Leaves & Decoction & Tea & $3 \mathrm{D}$ & \multirow{2}{*}{17} & \multirow{2}{*}{23} & \multirow{2}{*}{0.74} \\
\hline & & Bark & Extraction & Tea & 1D & & & \\
\hline \multirow{2}{*}{5} & \multirow{2}{*}{ Banaba } & Leaves & Decoction & Tea & 1D & \multirow{2}{*}{16} & \multirow{2}{*}{22} & \multirow{2}{*}{0.73} \\
\hline & & Flowers & Decoction & Tea & $1 \mathrm{D}$ & & & \\
\hline \multirow{2}{*}{6} & \multirow{2}{*}{ Okra } & Fruit & Decoction /Fresh & Tea/Viand & $3 \mathrm{D}$ & \multirow{2}{*}{14} & \multirow{2}{*}{21} & \multirow{2}{*}{0.67} \\
\hline & & Flowers & Decoction & Tea & 1D & & & \\
\hline 7 & Luya & Rhizomes & Decoction /Fresh & Tea & $2 \mathrm{D}$ & 13 & 20 & 0.65 \\
\hline \multirow{3}{*}{8} & & Leaves & Decoction & Tea & 1D & & & \\
\hline & Duhat & Fruit & Fresh & Eaten as raw & 1D & 12 & 19 & 0.63 \\
\hline & & Seeds & Extraction & Tea & 1D & & & \\
\hline 0 & Mangocton & Rinds & Decoction & Tea & $2 \mathrm{D}$ & 12 & 10 & 062 \\
\hline 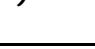 & Mansosecti & Fruit & Fresh & Eaten as raw & $3 \mathrm{D}$ & 12 & 19 & 0.03 \\
\hline 10 & Sabila & Leaves & Decoction & Tea & 1D & 10 & 15 & 0.62 \\
\hline 11 & Abukado & Leaves & Decoction & Tea & $2 \mathrm{D}$ & 9 & 15 & 060 \\
\hline 11 & ADukado & Fruit & Fresh & Eaten as raw & $2 \mathrm{D}$ & $y$ & 10 & 0.00 \\
\hline 12 & Akanulko & Leaves & Decoction & Tea & $1 \mathrm{D}$ & 8 & 14 & 0.57 \\
\hline 12 & Aйарutкu & Flowers & Decoction & Tea & 1D & 0 & 14 & \\
\hline 13 & Balbas pusa & Leaves & Decoction & Tea & $3 \mathrm{D}$ & 8 & 14 & 0.57 \\
\hline 14 & Basil & Leaves & Decoction & Tea & $2 \mathrm{D}$ & 7 & 13 & 0.53 \\
\hline 15 & Bawang & Cloves & Extraction & Tea & 1D & 7 & 13 & 0.53 \\
\hline 16 & Caimito & Leaves & Decoction & Tea & 1D & 6 & 12 & $0 . .50$ \\
\hline 17 & Dalanghita & Fruit & Fresh & Eaten as raw & $3 \mathrm{D}$ & 6 & 12 & 0.50 \\
\hline 18 & Kalingag & Bark & Extraction & Tea & $2 \mathrm{D}$ & 5 & 10 & 0.50 \\
\hline 19 & Kamatsile & Fruit & Fresh & Eaten as raw & $3 \mathrm{D}$ & 4 & 8 & 0.50 \\
\hline 20 & Kasuy & Bark & Extraction & Tea & $3 \mathrm{D}$ & 3 & 7 & 0.43 \\
\hline 21 & Lagundi & Leaves & Decoction & Tea & $1 \mathrm{D}$ & 3 & 7 & 0.43 \\
\hline 22 & Madder & Leaves & Decoction & Tea & $1 \mathrm{D}$ & 3 & 9 & 0.33 \\
\hline 23 & Pandan & Leaves & Decoction & Tea & $1 \mathrm{D}$ & 6 & 20 & 0.30 \\
\hline 24 & Saging & Flower & Cook & Viand & $2 D$ & 1 & 5 & 0.20 \\
\hline 25 & Sinta & Leaves & Decoction & Tea & 1D & 1 & 18 & 0.06 \\
\hline
\end{tabular}

a1D-once a day; 2D-twice a day; 3D-thrice a day

buse Value $=$ [the number of use reports cited by each respondent for a given plant specie $(U)$ /the total number of respondents interviewed for a given plant $(n)$. $\mathrm{UV}=\Sigma U / n$. 


\section{CONCLUSION}

Commonly used medicinal plants for treating diabetes are ampalaya (Momordica charantia Linn.), malunggay (Moringa oleifera Lam), guyabano (Annona muricata Linn.), guava (Psidium guajava Linn.), banaba (Lagerstroemia speciosa (L.) Pers.), okra (Hibiscus esculentis Linn.), luya (Amomum zingiber Linn.), duhat (Myrtus cumini Linn.), mangosteen (Garcinia mangostana Linn.), and sabila (Aloe vera Linn.). Occasionally utilized plants are abukado (Persea gratissima Gaertn.), akapulko (Cassia alata Linn.), balbas-pusa (Orthosiphon aristatus (Blume) Miq.), basil (Ocimum album Blanco), bawang (Allium sativum Linn.), caimito (Chrysophyllum cainito Linn.), dalanghita (Citrus madurrensis), kalingag (Cinamomum mercadoi Vidal), kamatsile (Pithecellobium dulce (Roxb.) Benth.), kasuy (Anacardium occidentale Linn.), lagundi (Vitex negundo Linn), madder (Rubia cordifolia Linn.), screw pine (Pandanus odoratissimus Linn. f.), saging (Musa paradisiaca Linn) and sinta (Andrographis paniculata (Burm. f.) Wall. ex Nees). Lastly, leaves are utilized for medicinal consumption and are prepared either by decoration or extraction and mostly are taken as a form of tea.

\section{ACKNOWLEDGMENT}

The author acknowledges ABCommunication 2Beve, ABEnglish 2A, BSChemistry 2, BSChemistry 3, BSES 2 and BSFT 1A (Academic Year 2014-2015) students for their help and assistance.

\section{REFERENCES}

1. Quisumbing E. Medicinal plants of the Philippines. Caloocan City: Katha Publishing, 1978.

2. Shanidar SR. Neahderthal flower burial in northern Iraq. Science 1975; 190:880-1.

3. Merrill ED. Flora of Manila Lehre, Codicote/ Herts. New York/N.Y, Cramer; Wheldon \& Wesley: Stechert-Hafner 1968.

4. Fabricant DS and Farnsworth NR. The Value of Plans Used in Traditional Medicine for Drug Discovery. Environ Health Perspect 2001; 109 (Suppl 1):69-75.

5. Schurz W. Leytle. The Manila Galleon 1939.

6. Philippine Health Statistics $2001-2010$, Philippines. Department of Health, Philippines. Available at http:// www.doh.gov.ph/kp/statistics/ selected_death.html. Accessed January 23 2015.

7. Shukla R, Sharma SB, Puri D, Prabhu KM and Murthy PS. Medicinal Plants for Treatment of Diabetes Mellitus. Indian J Clin Biochem 2000; 15(Suppl.); 169-177.

8. Bhushan MS, Rao CV, Ojha, SK, Vijayakumar M, Verma A. An Analytical Review of Plants for Antidiabetic Activity with their Phytoconstituent and Mechanism of Action. Int
J Pharm Sc Res 2010; 1:29-46

9. Population and Annual Growth Rates for the Philippines and Its Regions, Provinces, and Highly Urbanized Cities, Census and Housing Population. National Statistics Office, Philippines 2010 Accessed January 21, 2015.

10. Friedman J, Yaniv Z, Dafni A and Palewitch D. A Preliminary Classification of the Healing Potential of Medicinal Plants, Based on a Rational Analysis of an Ethnopharmacological Field Survey among Bedouins in the Negev Desert, Israel. J Ethnopharmacol 1986; 16:275 $-87$.

11. Phillips O, Gentry AH, Reynel C, Wilkin P, and Galvez-Durand CB. Quantitative Ethnobotany and Amazonian Conservation. Conserv Biol 1994; 225-48.

12. Merill ED. An Enumeration of Philippine Flowering Plants. Amsterdam: A. Asher 19671968.

13. Van Lerberghe W. The World Health Report 2008: Primary Health Care: Now More Than Ever. World Health Organization 2008.

14. Yeh GY, Eisenberg DM, Kaptchuk TJ and Phillips R. Systematic Review of Herbs and Dietary Supplements for Glycemic Control in Diabetes. Diabetes Care 2003; 26:1277-94.

15. Ezuruike UF and Prieto JM. The Use of Plants in the Traditional Management of Diabetes in Nigeria: Pharmacological and Toxicological considerations. J Ethnopharmacol 2014; 155:857-92.

16. Shukla R, Sharma SB, Puri D, Prabhu KM and Murthy PS. Medicinal Plants for Treatment of Diabetes Mellitus. Indian J Clin Biochem 2000; 15:(SuppL):169-77.

17. Alison MG, Abdel-Wahab AM and Flatt PR. The Traditional Plant Treatment, Sambucus nigra (elder), Exhibits Insulin- Like and Insulin -Releasing Actions In Vitro. J Nutr 2000; 130:15-20.

18. Farnsworth NM, Olayiwola A, Bingel AS, Djaja SS and Guo Z. Medicinal Plants in Therapy. Bull World Health Organ 1985; 63:965-81.

19. Erasto P, Adebola PO, Grierson DS and Afolayan AJ. An Ethnobotanical Study of Plants Used for the Treatment of Diabetes in the Eastern Cape Province, South Africa. Afr J Biotechnol 2005; 4:1458-60.

20. Modak M, Dixit P, Londhe J, Ghaskadbi S and Devasagayam TPA. Indian Herbs and Herbal Drugs Used for the Treatment of Diabetes. J Clin Biochem Nutr 2007; 40:163-73.

21. Grover JK, Yadav S, and Vats V. Medicinal Plants of India with Antidiabetic Potential. J Ethnopharmacol 2002; 81:81-100.

22. Dey L, Anoja SA, Yuan C-S. Alternative Therapies for Type 2 Diabetes. Alternative Med. Rev. 2002; 7:45-58.

23. Swanston-Flatt SK, Day C, Bailey CJ and Flatt PR. Traditional Plant Treatments for Diabetes. Studies in Normal and Streptozotocin Diabetic Mice. Diabetologia 1990; 33:462-4. 
24. World Health Organization. WHO traditional medicine strategy 2014-2023. 2013. Geneva: World Health Organization 2015.

25. De Padua LS, Lugod GC, Pancho JV. Handbook on Philippine medicinal plants. Technical Bulletin. 1977; 2(3). 
